\title{
SEGMENTATION TECHNIQUES IN X-RAY IMAGES FOR THE ANALYSIS OF MANGO STEM BORING INSECTS
}

\author{
TIMOTHY CROCKER, ROBINSON THAMBURAJ AND ATULYA K. NAGAR
}

\begin{abstract}
Mango plants are generally affected by various pests. The stem borer is a very harmful pest as its grub feeds on the internal parts of the tree leaving very little external evidence of damage to the host plant. Existing pest management methods suffer from the lack of knowledge about the precise location of the pest within the plant and the extent of internal damage. Recent laboratory experiments prove that these drawbacks can be overcome by using radiography as a means of study of the internal constituents of the plant's stem. This paper proposes a method to precisely segment the tree trunk followed by the segmentation of the stem boring insect's tunnels that appear in an X-ray image.
\end{abstract}

\section{INTRODUCTION}

Mangifera indica L. (the common mango) is a tropical fruit that is known to have been cultivated for over 4000 years in certain countries. It is believed that the fruit was originally cultivated in parts of South Asia and the production has spread throughout tropical and sub-tropical regions over the past few centuries. Natural hybridization has played an important role in the production of exotic varieties [1]. There is considerable trade of the fruit and associated products across the globe between the mango-producing countries and other regions. With the increase in production, consumption and trade, high trading standards have been imposed on the quality and quantity of the production of mango. In order to ensure good quality, cultivators of the fruit practice various conventional production methods and follow periodic pest management methods.

Mango is known to be effected by various pests all through its life-cycle. It has been recorded that a total of 476 arthropods, 19 nematodes, 62 fungi, 5 bacteria, one virus and one alga affect the plant [2]. The stem boring insect of mango (Batocera rufomaculata) is a harmful insect pest. It does not appear in all mango cultivating regions, but in the places where it does, it causes severe damage and loss. The damage is caused by the grub of the insect that tunnels upward in the stem of the plant. If this pest is not detected during its early instars, the damage done by the late instars of the pest can prove fatal to the tree. Current methods in managing this pest include the usage of chemicals or the cutting down of infected trees. Chemical treatment is useful only during the early stages of infestation. The felling of infected branches or the entire tree is done in the worst

$M S C$ (2010): primary 68U10.

Keywords: segmentation, Batocera rufomaculata, Mangifera indica, X-ray, pest management. 
case of infestation, resulting in environmental damage and economic loss. The application of pesticides is done only at the entrance and exit holes where the external damage is visible. Branches or trees are cut only when they appear to wilt. In certain cases, even healthy and un-infested branches are cut as a measure of precaution. All this is done without having any knowledge of the locality of the pest and the extent to which the damage is caused by the pest within the plant.

The use of radiography in the field of medical imaging has gained large scope in the recent past with the introduction of highly improved imaging techniques. Some of the most common radiographic scanning methods include X-rays and Computed Tomography (CT). These methods have led to rapid advancements in the healthcare domain. The use of radiography is also seen in many other streams such as industrial construction, quality testing, security, etc. However, the use of radiography in fields such as pest management has been restricted greatly due to the lack of in situ means of these imaging tools.

\section{BACKGROUND}

\subsection{Biology and symptoms of mango stem borer attack}

The mango stem borer is a large long-horned grayish beetle. Adult beetles measure up to 50 millimeters in length. Eggs are laid by females in cracks along the bark of a tree. Damaged trees or branches are more susceptible to attack. The incubation period extends for about 10 to 15 days. The newly hatched larvae eat horizontally inward, into the sapwood. The plant tends to heal over time and the entrance holes of the larvae are reduced in size. Larvae then develop within the plant by feeding on the cortex as shown in Figure 1. This internal feeding results in the formation of tunnels within the stem and causes structural weakening and reduction of the flow of sap within the plant. Once the pupae reach maturation, they bore horizontally

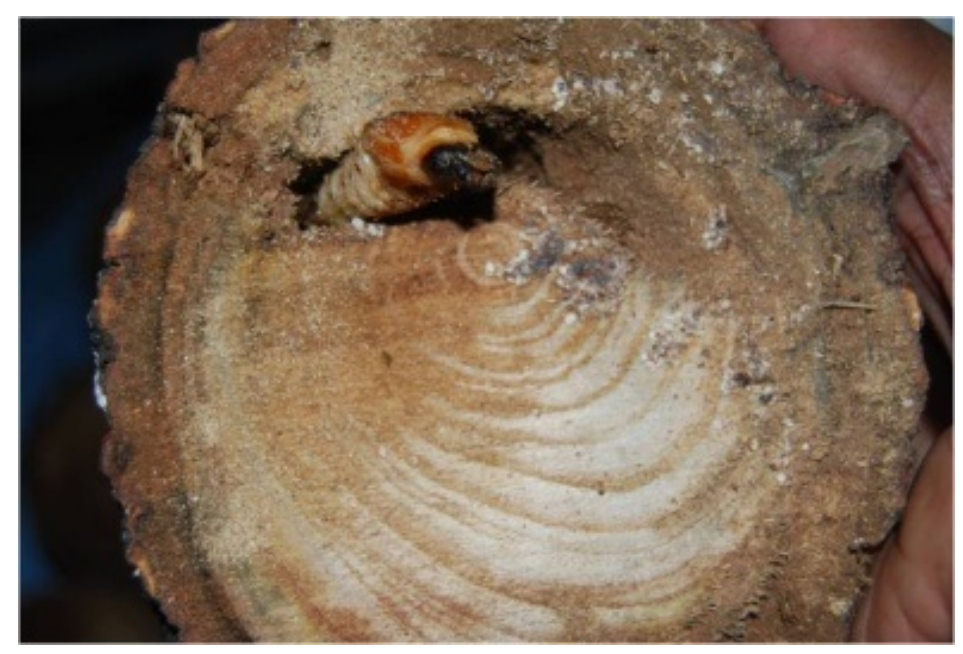

Figure 1. Cross-section of mango stem showing the grub of the stem borer insect (Batocera rufomaculata). 
outward through the bark and adult beetles emerge. The exit holes are around 13 to 15 millimeters in width. There is only one generation of the pest per year [3].

Most of the damage that is caused by the mango stem borer is internal to the plant. However, there are certain external symptoms that clearly indicate the presence of the pest on a given tree. These include the presence of adult beetles on the tree, entrance and exit holes on the bark of the tree (Figure 2), the wilting of branches or the entire tree, the oozing of sap from the entrance and exit holes and the presence of frass (a material like saw dust that is excreted by the feeding larvae as shown in Figure 3) that is found at the holes in the bark or at bottom of the tree [4].

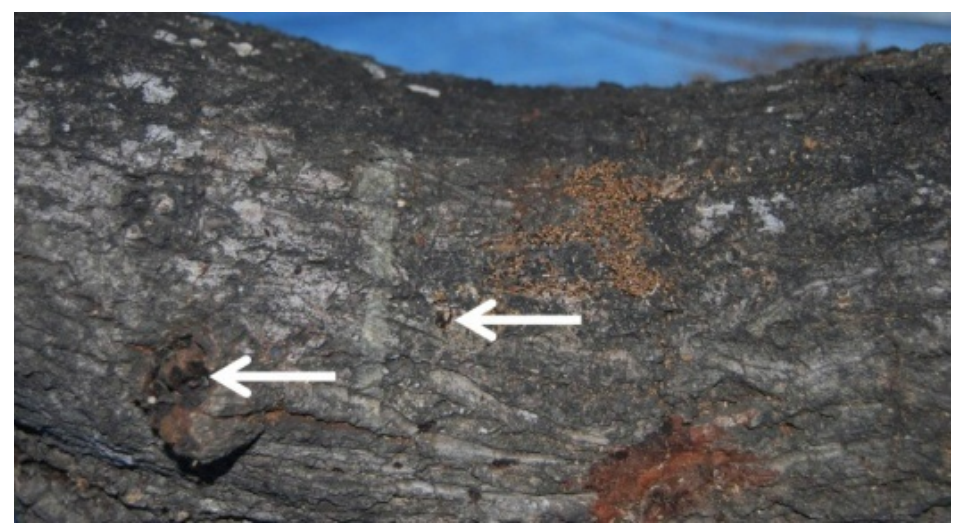

Figure 2. Small entrance holes seen along the bark of the tree.

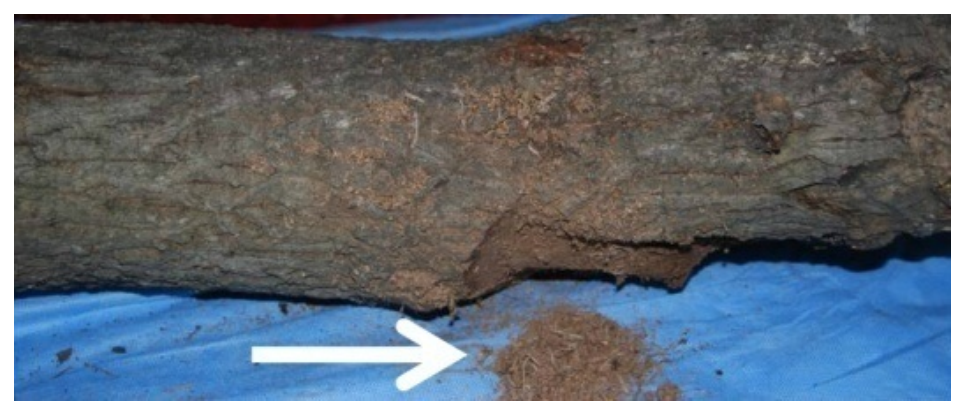

Figure 3. Frass excreted by the larvae as a result of feeding on the sapwood.

\subsection{Pest distribution and recent outbreak}

The mango stem borer also affects other host plants such as cashew nut, jackfruit, fig, rubber and mulberry. Certain varieties of mango have been found to be more susceptible to the stem borer attack. The pest is found to be distributed globally in countries such as Pakistan, India, Ceylon, Andaman, China (Tibet, Hainan), Nepal, Myanmar, Thailand, Laos, and Malaysia. It is said to have been introduced in Syria, Jordan, Lebanon, Israel, Africa, Madagascar, Comoros, Mauritius, Virgin 
Islands, and Puerto Rico. In India, the pest has been recorded in the states of Kashmir, Punjab, Uttar Pradesh, Assam, Bihar, Madhya Pradesh, Maharashtra, Andhra Pradesh, Karnataka, Tamil Nadu and Kerala [5].

In the year 2012, the damage caused by the mango stem borer was reported in Kolar District, Karnataka, India. The report highlighted the fact that the Badami variety of mango was mostly under attack by the pest because of the sweetness and taste of the core of the tree trunk [6].

\subsection{Existing pest management methods and drawbacks}

The existing practices in managing the stem borer menace is to identify the trees affected by the pest and physically remove adult beetles that live on the tree. The grubs of the insect which live within the tree, that can be reached using a flexible wire are also physically removed or killed. Chemical treatment is used to kill the larvae that cannot be physically reached. However, if the insect has reached the interior parts of the stem, the chemical treatment is ineffective. Also the application of pesticides results in the accumulation of chemicals in the aerial parts of the plant, such as the fruit, and makes it unfit for consumption. Branches and trees that are severely affected are cut down. In certain cases, all the trees in an infested region are cut down as a measure of containing the pest. All these pest management methods are done without the knowledge of the exact location of the grub within the plant and with no perception of the actual extent of damage within the plant.

\subsection{Stem Borer Investigation}

A sample of four mango stems that showed external symptoms of stem borer infestation were cut and taken for examination. The internal damage of the stems was clearly visible from the X-ray analysis performed. One of the branches that showed extensive internal damage, as shown in Figure 4, was chosen for further analysis. The log that was selected for further examination was X-rayed along the vertical axis from various angles. The X-ray images are shown in Figure 5. The images obtained from these tests are used for image analysis.

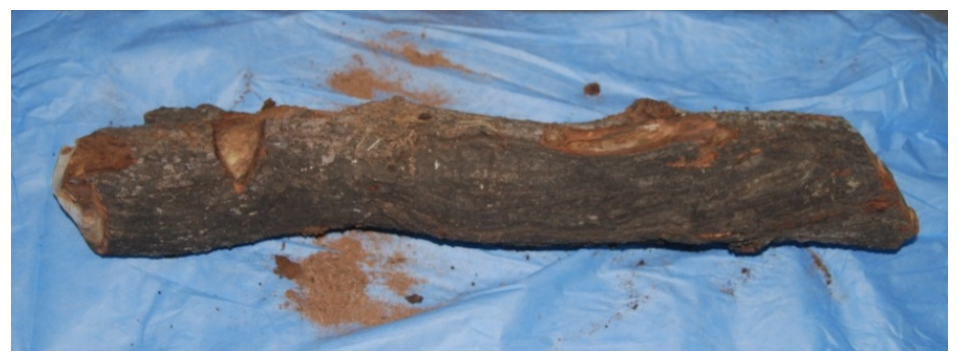

Figure 4. Mango stem taken for analysis of stem borer infestation.

X-ray images are found to be projections of three dimensional objects onto a two dimensional plane. It may also be described as a superimposition of shadows of overlapping objects onto a photo-sensitive plane. An X-ray image is formed as 
a result of the different attenuation levels of the objects being X-rayed. Attenuation may be defined as the reduction in the intensity of X-rays as they pass through objects of various densities. A less dense medium such as air appears black on an $\mathrm{X}$-ray film while a denser medium such as a bone, wood or metal appears white.
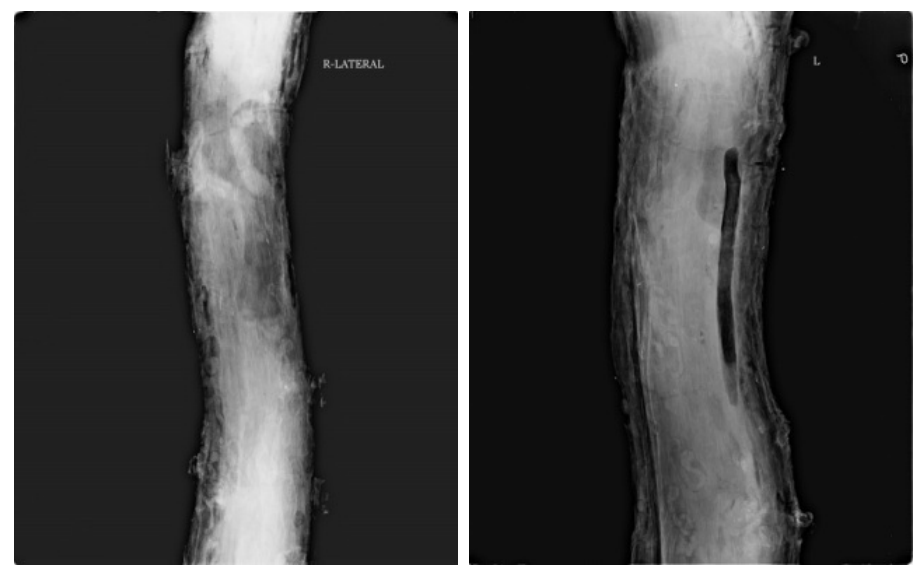

Figure 5. X-ray images taken along the vertical axis of one of the mango stems chosen for investigation.

\section{Purpose}

The aim of this study is to use radiography as a means of ascertaining the internal damage caused to a tree by stem boring larvae. X-ray images expose the exact location of the larvae within the plant. The tunneling structures created by the pests are also visible in an X-ray of the bark. Integrated pest management systems can use this information to precisely detect the location, count, amount of infestation, etc, within a plant. The first step in digital image analysis on these $\mathrm{X}$-ray images is to segment the stem of the plant precisely so that this area can be used as the region of interest within which further analysis can be performed. The accurate segmentation of the stem region in an X-ray image is significant, as it reduces the total area in the image that is processed to a more specific and smaller region. Using this region the tunnels within the stem can be segmented. Tunnels appear as elongated tubular regions within the stem that are easily distinguishable from the stem by a distinct change in intensity values. The segmentation of the plant stem followed by the segmentation of the tunnels within the stem is described below.

The segmentation of the stem was initially attempted using the Otsu's thresholding method [10]. However, the resulting binary images showed the stem region to have several holes and a very irregular boundary. This was mainly due to the abrupt changes in intensity found throughout the region of interest. The use of edge detection algorithms resulted in several undesired edges being obtained within the stem region. Hence a combinatorial approach to this problem was developed 
using a few of the existing image processing techniques such as thresholding and morphology.

\section{Methodology}

\subsection{Segmentation of the stem}

As an initial step towards identifying the constituents of the stem, the region of interest is first segmented. That is, the stem of the tree is identified and differentiated from the background. The use of thresholding followed by morphological operations did not produce satisfactory results. Hence an algorithm with a dual approach was created. The two main steps involved in this algorithm are: i) to identify the boundary line of the stem and ii) to identify the region with lesser intensity in the image, which is actually the stem of the plant. The first step focuses on defining an outline, while the second focuses on defining a region. The defects brought about by one approach are overcome by the other.

Preprocessing. The X-ray images used in this study were found to have a small amount of salt and pepper noise in certain regions, and also a repeating pattern as a part of the background of the image. This pattern appeared as horizontal lines with very small changes in intensity values when compared to the background. In order to remove the noise in the image and make the background intensity more uniform, X-ray images were improved before the segmentation process using the median filter [7] as shown in Figure 7.

Observation. In the X-ray images that were taken, it was noted that the entire stem of the plant was outlined by a clearly darker region. On analysis it was found that this region could be clearly segmented from the image by progressively thresholding the image and stopping at a critical threshold value. The method used to calculate this value is described below. The following step may be seen as the first step towards identifying the boundary of the stem.

Calculation of critical threshold. For every gray value $v$, starting from 0 to 254, two binary images were obtained by thresholding, with threshold values $v$ and $v+1$. The number of white pixels in each of the resulting binary images was calculated and the difference was taken. At a certain threshold the difference increased drastically, and this value was taken as the critical threshold. At this point the iterative process was stopped. The graph in Figure 6 shows the decrease in the number of white pixels in the resulting binary image as the threshold value increases.

The X-ray image that was processed to obtain this graph is shown in Figure 7 . From the graph, a clear drop in the number of white pixels in the binary image is seen at the threshold value 14. This threshold value is taken to be the critical threshold point at which the border of the tree's stem can be clearly and easily segmented from the image. The difference in the binary images, before and after the critical threshold value is clearly seen in Figure $8 \mathrm{a}$ ) and $8 \mathrm{~b}$ ). 


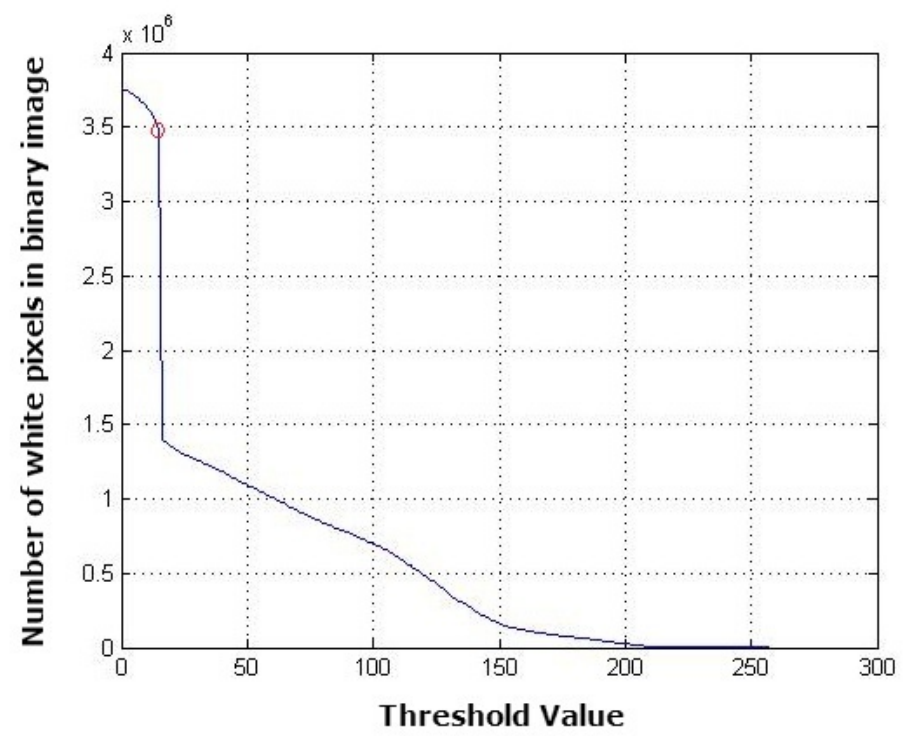

Figure 6. Graph for calculating the critical threshold - the point after which there is a significant decrease in the number of white pixels in the resulting binary image.

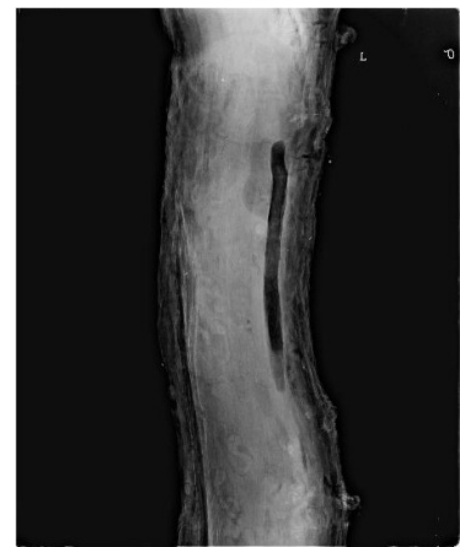

Figure 7. Application of median filter.

Morphology. A series of morphological operations are performed on the inverted binary image, which is obtained after the calculation of the critical threshold value. The order of the morphological processes performed is as follows. First, the morphological close operation [8] is performed. This operation is done twice. Each time a different structuring element is used. The first time, a rectangular structuring element is used. The rectangle is defined such that the height is greater than the width. Based on the image size, a 10x20 matrix is defined as the rectangular structuring element. This is done with the intention of making the segmented white regions vertically continuous. For the second morphological close, a disk 
shaped structuring element of radius 10 is used to ensure that the segmented white regions are horizontally continuous. These two structuring elements were chosen based on the shape of the outline of the tree's stem that is vertically elongated, and horizontally narrower. The result of this step is shown in Figure 9 a).
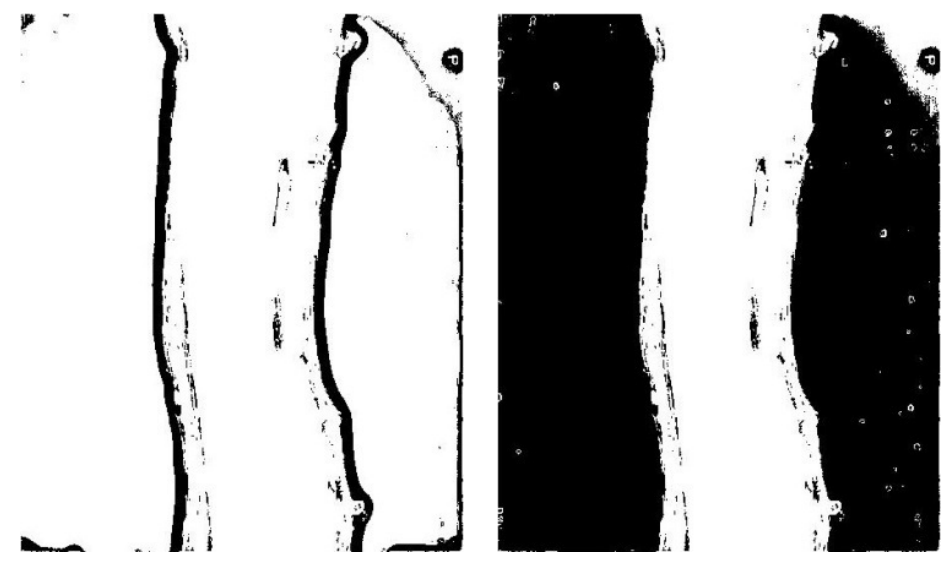

Figure 8. a) Binary image at threshold value 14, b) binary image at threshold value 15 .

Following the morphological closing operations, the smaller blobs were removed as shown in Figure $9 \mathrm{~b}$ ). This process removes most of the unwanted regions within the image. However, there may be certain larger regions that are undesired and appear along the edge of the image. These artifacts will be handled later. After removing the smaller objects, the white region may contain holes as a result of the morphological closing performed earlier. Hence the holes are removed so that the tree's outline can be obtained more precisely. This is accomplished by performing a morphological thinning operation [9] as shown in Figure 10 a). This process reduces the width of the segmented blobs to a continuous linear skeleton like structure.
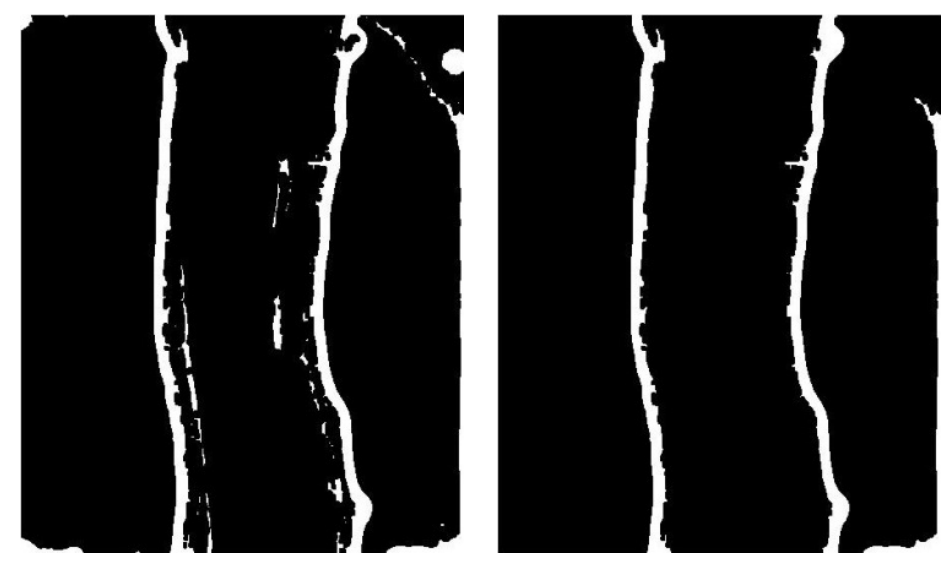

Figure 9. a) Morphological close using rectangular and disk shaped structuring element,

b) removal of smaller blobs. 
Defects of the thinned outlines. There are three defects that are found with the thinned outline image that is obtained after applying morphology. The first is that the thinning algorithm produces branches based on the shape of the white regions. If these branches are removed, the region of interest (the stem) can be more precisely defined. The second is the presence of undesired outlines in the image resulting from the presence of artifacts that have not been removed by earlier steps. The third defect is that the thinned lines do not extend up to the border of the image. This means that outline of the stem is not complete and the region of interest cannot be well defined. In order to deal with these defects, the following steps are used.
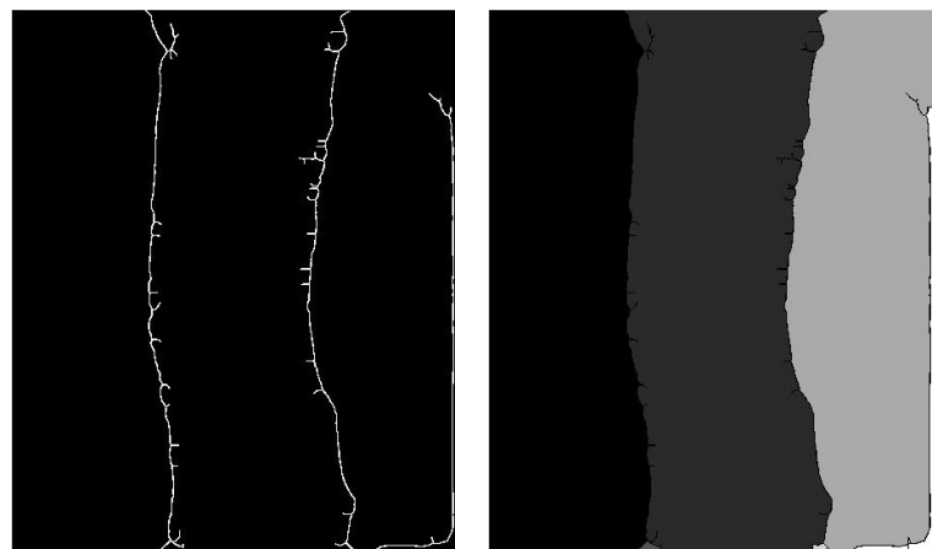

Figure 10. a) Morphological thinning, b) labeled image showing separated regions.

Completion of stem outline. The third defect is dealt with first. The dilation of the thinned lines did not help in making the tree's outline complete up to the border of the image. Hence, another method was adopted using the end points of the thinned lines. An end point may be defined as any white pixel that has only one neighboring white pixel specific to an 8-neighborhood connectivity. Having obtained the endpoints, a filtering process was carried out on these points in order to find only the end points that were near any one of the four borders of the image. This was done by finding the distance between the coordinates of the pixel and the boundaries of the image. If the end points were found to be within a distance of 50 pixels to one of the image borders, they were taken for further consideration. The filtered end points were then connected to the image boundary either horizontally or vertically based on their closeness to a corresponding boundary. The connection was made by making all the pixels in the line between the end point and the nearest boundary white, thereby completing the tree's outline. At the completion of this step, the image is divided into well defined regions that are bounded by the thin lines. This binary image is inverted and then labeled for all the pixels in any specific region to have the same label as shown in Figure $10 \mathrm{~b}$ ). The labeled image will be used in the next process.

Otsu's thresholding. In order to overcome the other two remaining defects of the thinned lines, an alternate approach is used, without trying to modify the 
thinned lines. This step can be taken for the second phase in the overall process as it uses the original grey image that was obtained after preprocessing. A binary image is obtained from this grey image by using the Otsu's threshold [10] value as shown in Figure $11 \mathrm{a}$ ). Using this binary image and the labeled image obtained in one of the pervious steps, the number of white pixels in each of the well defined regions is calculated. The region that has the maximum number of white pixels is treated as the stem region. By this inference, all the other regions can be treated as background regions and thereby overcoming the second defect of the thinned lines described earlier, which was due to the presence of undesired edges.
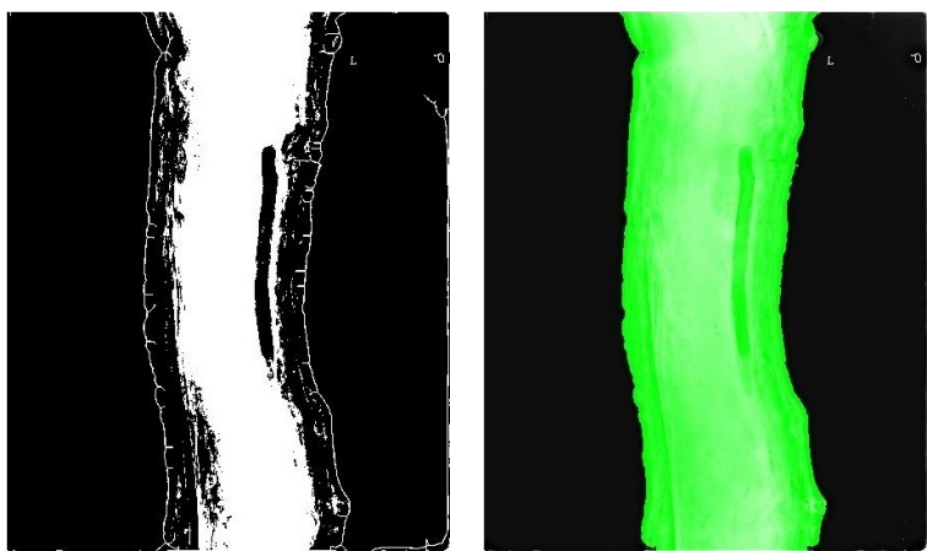

Figure 11. a) Binary image using Otsu's threshold along with region boundaries, b)

segmentation of stem.

Refining the region of interest using morphology. As a final step in refining the obtained region of interest, the first defect introduced as a result of line thinning has to be removed. The undesired branches of the thinned lines that appear within the region of interest are removed by performing a morphological close operation using a disk shaped structuring element. The resulting region may have holes in it due to the morphological close. Hence, the holes are filled, and the resulting blob is taken for the segmented stem of the plant as shown in Figure $11 \mathrm{~b}$ ).

\subsection{Segmentation of insect tunnels within the stem}

The segmentation of the tunnels is based on the fact that the tunnels appear as darker regions within the stem. This is because they contain air or frass which is a less dense substance than wood. Therefore, as a primitive step in segmenting hollow regions in the stem, all the darker regions that were enclosed completely within the segmented stem region, were considered tunnels. The result of the segmentation of tunnels within the stem is shown in Figure 12.

\section{Results}

The following images were obtained as results of the various steps in the method described above. Figure 13 a) shows the X-ray image of a mango stem that does 
not contain any tunnels created by the stem boring insects. Figure $13 \mathrm{~b}$ ) shows the segmentation result of the same.

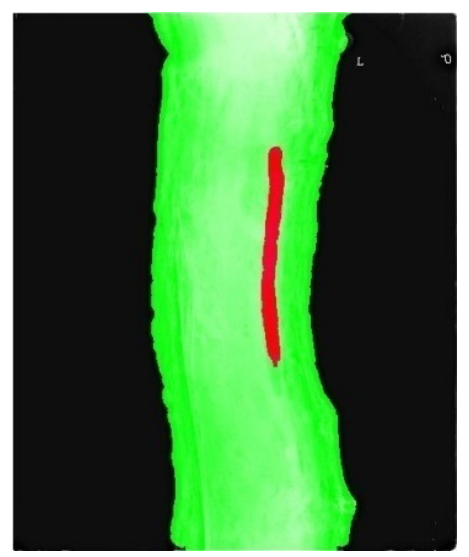

Figure 12. Segmentation of tunnel within the stem.
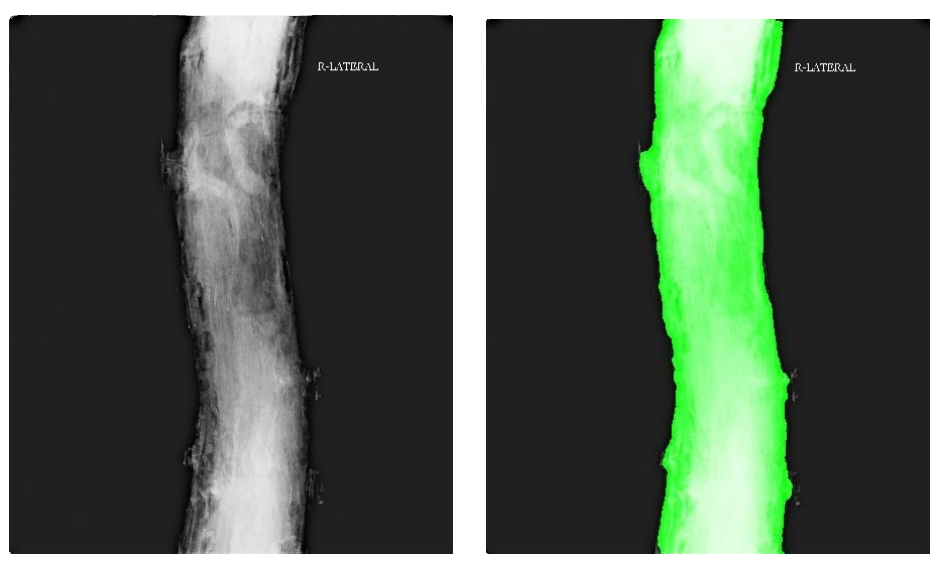

Figure 13. a) Sample X-ray image of stem without insect tunnel, b) segmentation result.

\section{Conclusion}

The stem of the plant was segmented precisely by using the dual approach. The first step was to divide the image into regions by taking the thinned boundary and the second was to find the most likely region that would be the stem. This method proved better than other algorithms in that the border of the stem appeared continuous and not broken. The problem of darker regions along the bark of the stem that appear in the binary image of the Otsu's thresholding was overcome by first finding the stems boundaries. The problems posed by the thinned region boundaries were overcome using the Otsu's thresholding method. The insect tunnels within the stem were also segmented. The algorithm for segmenting the tunnels needs to be improved to handle more complexly structured tunnels. 
With the development of an automated image analysis system for the identification of stem borer pests, integrated pest management techniques can be devised based on the X-rays analysis. The extent of internal damage to the plant can be ascertained and more specific treatment in terms of locality can be administered. If the stem borer attack can be identified and prevented at the initial stage with the precise knowledge of the presence of the pest within the plant, greater damage can be averted in terms of environmental damage and economic loss.

Acknowledgement. The authors would like to thank Dr. D. Janardhana Reddy, Ekavera Agro Farm, Chennai, India, and Dr. Narashiman, Department of Botany, Madras Christian College, Chennai, India, for providing the images that are being used for this study.

\section{REFERENCES}

[1] The Wealth of India, Raw Materials, Vol. VI (L-M), Council of Scientific and Industrial Research, New Delhi, 265-284.

[2] Mangoes from India, Draft Revised Import Policy, Department of Agriculture, Fisheries and Forestry, Australian Government, July 2004, 29-31.

[3] Agropedia Website: http://www.agropedia.iitk.ac.in, Retrieved October 3rd, 2013.

[4] Tamil Nadu Agricultural University (TNAU) Agritech Portal Website: http://www.agritech.tnau.ac.in, Retrieved October 15th, 2013.

[5] Plantwise Knowledge Bank Website: http://www.plantwise.org, Retrieved October 3rd, 2013.

[6] Deccan Herald Website: http://www.deccanherald.com, Retrieved October 3rd, 2013.

[7] J.S. Lim, Two-Dimensional Signal and Image Processing, Prentice Hall, Englewood Cliffs, NJ, 1990.

[8] P. Soille, Morphological Image Analysis, 2nd ed., Springer, New York, 2003.

[9] R. C. Gonzalez and R. E. Woods, Digital Image Processing, 3rd ed., Prentice Hall, NJ, 2008.

[10] N. Otsu, A threshold selection method from gray-level histograms, IEEE T. Syst. Man Cy. 9 (1979), 62-66.

Timothy Crocker, Department of Mathematics, Madras Christian College, Chennai - 600059 , India

e-mail: tjc91189@gmail.com

Robinson Thamburaj, Department of Mathematics, Madras Christian College, Tambaram, Chennai - 600059 , India

e-mail: robin.mcc@gmail.com

Atulya K. Nagar, Department of Mathematics and Computer Science, Liverpool Hope University, Hope Park, Liverpool L16 9JD, UK

e-mail: nagara@hope.ac.uk 